\title{
The eight-item Morisky Medication Adherence Scale MMAS: Translation and validation of the Malaysian version
}

\author{
Harith Kh. Al-Qazaz ${ }^{a}$, Mohamed A. Hassalib, ${ }^{*}$, Asrul A. Shafie ${ }^{b}$, Syed A. Sulaimana, \\ Shameni Sundram ${ }^{c}$, and Donald E. Morisky ${ }^{d}$ \\ a Discipline of Clinical Pharmacy, School of Pharmaceutical Sciences, Universiti Sains Malaysia, \\ 11800 Penang, Malaysia \\ b Discipline of Social and Administrative Pharmacy, School of Pharmaceutical Sciences, \\ Universiti Sains Malaysia, 11800 Penang, Malaysia \\ c Hospital Balik Pulau, Balik Pulau, 11000 Penang, Malaysia \\ ${ }^{d}$ Department of Community Health Sciences, UCLA School of Public Health, 650 Charles E. \\ Young Drive South, Los Angeles, CA, USA
}

\begin{abstract}
Aims-To translate and examine the psychometric properties of the Malaysian version of the Morisky Medication Adherence Scale (MMAS) among patients with type 2 diabetes.

Methods-A standard "forward-backward" procedure was used to translate MMAS into Malay language. It was later validated on a convenience sample of 223 type 2 diabetes outpatients between May and September 2009. Reliability was tested for internal consistency. Validity was confirmed using convergent and known group validity.
\end{abstract}

Results-Employing the recommended scoring method, the mean \pm SD of MMAS scores was $6.13 \pm 1.72$. Moderate internal consistency was found (Cronbach's $\alpha=0.675$ ), the test-retest reliability value was $0.816(p<0.001)$. A positive correlation between the eight- and four-item MMAS was found $(r=0.792 ; p<0.01)$. A significant relationship between MMAS categories and HbA1c categories $\left(\chi^{2}=20.261 ; p \geq 0.001\right)$ was found. The MMAS sensitivity and specificity, with positive and negative predictive values were $77.61 \%, 45.37 \%, 46.84 \%$ and $76.56 \%$, respectively.

Conclusions-The findings of this validation study indicate that the Malaysian version of the MMAS is a reliable and valid measure of medication adherence which can now be used.

\section{Keywords}

Type 2 diabetes; Adherence; Validation; Morisky scale

\section{Introduction}

Diabetes mellitus (DM) is a growing health problem with a continuously increasing prevalence and high levels of morbidity and mortality. The International Diabetes Federation has predicted that there will be 380 million individuals with diabetes in 2025

\footnotetext{
(C) 2010 Elsevier Ireland Ltd. All rights reserved.

*Corresponding author. Tel.: +60 124421605; fax: +60 46570017. azmihassali@usm.my (M.A. Hassali).

Conflicts of interest

There are no conflicts of interest.
} 
compared with an estimated 240 million individuals in 2007, with a higher disease burden in low- and middle-income nations [1]. In Malaysia, the Third National Health and Morbidity Survey (3rd NHMS) showed that the prevalence of the type 2 DM for adults aged 30 years old and above now stands at a staggering $14.9 \%$, with highest prevalence among those of the Indian ethnicity at $19.9 \%$ [2]. Effective and successful glucose control requires the use of effective medications over time. The morbidity and mortality resulting from micro- and macro-vascular complications of type $2 \mathrm{DM}$, place a considerable financial burden on individual patients and on society $[3,4]$.

Although there are many factors that affect glycemic control among patients with diabetes, it is known to be improved by adherence to DM medications, and treatment utility is limited by lack of adherence [5-7]. According to the World Health Organization, adherence is defined as "the extent to which a person's behaviour - taking medication, following a diet and/or executing lifestyle changes - corresponds with agreed recommendations from the health care provider" [8]. Several studies on medication adherence have concluded that adherence among type 2 DM patients is unsatisfactory (36-93\%) [5,7]. A systematic review by Carmer confirmed that many patients with diabetes took less than the prescribed amounts of their medications [9]. Inadequate adherence compromises safety and treatment effectiveness, leading to increased mortality and morbidity with considerable direct and indirect costs to the healthcare system $[10,11]$.

Different tools have been used to evaluate and assess patients' adherence to medication, and there is no "gold standard" measure of medication adherence [12]. Standardized, selfreported questionnaires have frequently been used because they are low in both cost and time expenditure. Early studies found that the self-report method was underestimating nonadherence when compared with pill counts or biological assays [13,14]. However, subsequent research suggests that the self-report method may provide a reasonably accurate estimate of adherence $[15,16]$. Among structured, self-reported scales, a four-item selfreported questionnaire to assess medication adherence was developed by Morisky et al. [17]. Although the four-item scale has shown poor psychometric properties, it is one of the most widely used self-reported measures of medications adherence [18]. Recently, an eight-item self-reported scale has been developed by Morisky et al. and called the Morisky Medication Adherence Scale (MMAS) [19]. Because of the wide use of the scale as a medication adherence measurement tool, we were interested in translating the MMAS and documenting some of its psychometric properties in Malaysian people with type 2 DM.

\section{Materials and methods}

\subsection{Ethics}

This study received approval from the Ministry of Health Ethical Committee MREC and approval from the General Hospital Penang. All subjects provided written consent before participation.

\subsection{Participants and setting}

Cross-sectional study design and methodology was used to elaborate the study data. This study was conducted in the Diabetes Clinic of the Penang General Hospital, Penang, Malaysia. Penang General Hospital is the largest public and tertiary hospital in Penang state. It provides health care, emergency treatment for all illnesses and accidents, primary and advanced medical healthcare. A target sample size of 100 patients was estimated to give a good precision for reliability and validity study [20]. Double sample size was estimated to overcome the erroneous results and to increase the reliability of the conclusion. A convenience sample of 223 type 2 diabetic outpatients was identified between May and 
September 2009. To be included in the study, patients had to (1) have been diagnosed with type 2 diabetes at least one year before, (2) use anti-diabetes medications, (3) be over 30 years of age, and (4) be able to communicate in the Malaysian language. Patients who had severe health problems or cognitive impairments and could not complete interviews were excluded. Face-to-face interviews included administration of the translated eight-item MMAS, followed by the original four-item Morisky scale, and collection of sociodemographic data. A total of 175 patients were eligible and included in the analysis (approximately $78.4 \%$ ). On the same day as the interviews, medical records were reviewed for haemoglobin $\mathrm{A} 1 \mathrm{C}(\mathrm{HbA1c})$ levels, number of hypoglycaemic medications, whether or not the patient used insulin, and the presence of diabetic complications such as neuropathy, retinopathy, nephropathy, or cardiovascular disease. In addition, about one-fifth of this sample $(n=44)$ was randomly selected and agreed for a one-month reliability test-retest analysis. Only 39 patients completed the test-retest after one month. All 175 face-to-face and 39 re-interviews were performed by the investigator, who is a pharmacist.

\subsection{Instrument translation}

The instrument consisted of three parts: part one collects socio-demographic data, part two is the eight-item MMAS and part three is the four-item original Morisky scale. Each item in the MMAS measures a specific behaviour and is not a determinant of adherence behaviour [19]. The three parts of the data collection sheet were translated together according to the international guidelines [21,22] as follows:

1. Forward translation of the original questionnaire was undertaken by translation from English to the Malay language to produce a version that was semantically and conceptually as close as possible to the original questionnaire. Translation was done by two qualified independent linguistic translators; both native speakers of Malaysian and proficient in English. Each translator produced a forward translation of the original questionnaire into the target language without any mutual consultation. One of the researchers, who is Malaysian, reviewed the two primary versions and compared them with the original.

2. Reverse translation from Malaysian to English was carried out by another translator, after repeated discussion between the translators and Malaysian researchers. Inconsistencies were resolved in a consensus meeting and a final version, ready for testing, was generated.

3. The translated questionnaire was distributed to 20 Malaysian patients who completed the questionnaire and commented on the questions. These individuals were not included in the study. The patients' comments were discussed by the researchers. Subsequently, six postgraduate pharmacy students who are experts in the field judged the face and content validity of the questionnaire.

4. The final version of the Malaysian questionnaire was completed and made available for the reliability and validity study. The questionnaire takes about 10-12 min to complete.

\subsection{Statistical analysis}

Descriptive statistics were used to describe demographic and disease characteristics of the patients and their medication adherences scores. Percentages and frequencies were used for the categorical variables, while means and standard deviations were calculated for the continuous variables. The characteristics of the whole sample and of the adherent groups were presented. The Chi square $\left(\chi^{2}\right)$ test was employed for categorical variables and analysis of variance (ANOVA) tests were used to evaluate the differences between the adherent groups. Internal consistency was assessed using Cronbach's alpha, and Spearman's rank 
correlation was used to assess test-retest reliability. The criterion for accepting Cronbach's alpha is a score above $0.5[23,24]$. Convergent validity was assessed using Spearman rank correlation between MMAS scores and the scores on the four-item, original Morisky scale. Correlations were interpreted using the following criteria: $0-0.25=$ little or no correlation, $0.25-0.5=$ fair correlation, $0.5-0.75=$ moderate to good correlation and greater than $0.75=$ very good to excellent correlation [25]. Known group validity was assessed through the association of HbA1c levels ( $\geq 7 \%$ and $<7 \%$ ) and MMAS categories using Chi square $\left(\chi^{2}\right)$ tests. Known group validity was also assessed by using $t$-test, assuming that patients with poor diabetes control also report lower levels of adherence. All analyses were performed using SPSS version 15.0 (SPSS Inc., Chicago, IL). The significance level was set at $p$ less than 0.05 .

\section{Results}

\subsection{Clinical and demographic data}

The final analysis included 175 patients with diabetes, with 39 patients completing the testretest portion. The characteristics of the total and adherent groups are shown in Table 1. Of the 175 patients with diabetes, $67(38.2 \%), 78(44.5 \%)$ and $30(17.1 \%)$ were in the low, medium and high adherence groups. Significant differences were found in educational levels, HbA1c levels, number of medications per day, MMAS scores, and four-item original MAS scores among the three groups $(p<0.05)$. No significant differences $(p \geq 0.05)$ were found among the three groups in terms of age, sex, BMI, race, employment and duration of diabetes.

\subsection{Reliability}

Cronbach's alpha test of internal consistency was 0.675 for the eight items in MMAS, which is below 0.7 but higher than 0.5 [23,24]. Its item to total correlation coefficient ranged from 0.287 to 0.459 (Table 2). The test-retest reliability of eight items MMAS indicates excellent reliability and stability of the instrument with Spearman's rank correlation coefficient of $0.816(p<0.001)$.

\subsection{Convergent validity}

A positive correlation between the MMAS and the four-item original MAS was found $(r=$ $0.792 ; p<0.01)$. The high correlation result indicates good to excellent correlation between the two scores [25].

\subsection{Known groups validity}

The Chi square $\left(\chi^{2}\right)$ test shows a significant relationship between MMAS categories and HbA1c categories $\left(\chi^{2}=20.261 ; p \geq 0.001\right)$. Around $77.6 \%$ of the low adherence patients were in the poor glycemic control group, while $73.3 \%$ of those in the high adherence group were in the good glycemic control group. Table 3 shows the distribution of the three adherence groups according to poor and good $\mathrm{HbA1c}$ level. As hypothesized, patients who reported poor control of their diabetes (HbA1c > 7\%) also reported lower levels of adherence to medications mean (SD) scores of MMAS for patients with poor diabetic control vs. good diabetic control group were 5.75 (1.51) and 6.79 (1.73), respectively, with $t$ $=3.982, p<0.001)$. The Spearman Rank Order Correlation coefficient between HbA1c and total score of MMAS was $-0.431(p<0.001)$, indicating a medium inverse association between MMAS scores and HbA1c. 


\subsection{Sensitivity and specificity}

To determine how good the MMAS perform to identify patients with poor glycemic control and to provide information in clinical practice, sensitivity and specificity were evaluated. For the sensitivity and specificity analysis, only two groups of adherence scores were used - low adherence patients as one group, and medium and high adherence patients together as the second group [19]. As shown in Table 3, the MMAS sensitivity and specificity were $77.61 \%$ and $45.37 \%$, respectively. The positive and negative predictive values were $46.84 \%$ and $76.56 \%$, respectively.

\section{Discussion}

The main objective of this paper was to report the reliability and validity of the translated version of the MMAS in a sample of patients with diabetes. This study was the first to systematically translate and validate the eight-item MMAS into the Malaysian language. Other studies evaluate the eight-item MMAS in diabetes patients in Thailand [26] and in HIV-positive patients in Sweden [27]. The original eight-item MMAS was tested by Morisky et al. [19] on a sample of hypertensive patients, and it was found that the scale was reliable with good predictive validity and sensitivity. The Thailand study showed that the eight-item MMAS had good convergent validity with good test-retest reliability in Thai patients with type 2 diabetes, but with poor sensitivity [26]. Our study among Malaysian patients with diabetes showed that the eight-item MMAS had good test-retest reliability (0.816) with good convergent validity and a sensitivity (77.61\%) but lower specificity $(45.37 \%)$. In terms of internal consistency and test-retest reliability, it is different from that of Morisky et al. [19], but consistent with the Thailand study [26]. Our sample of patients was smaller than the sample used in both previous studies, and this might explain the differences from the Morisky et al. study in terms of the alpha values [19] as the internal consistency is actually a correlation coefficient and the sample size can affect the result [28]. Another explanation of the lower alpha value is that most of the items on the scale use a binary response (yes/no), and if more response choices applied like the five-level Likert's scale, the alpha value would be improved because the lower measurement error is associated with more response options [29]. For the convergent validity, a high correlation $(r=0.792)$ indicates that the translated eight-item MMAS correlates well with the translation of the previous four-item Morisky scale, and this improved convergent validity. High correlation between the eight-item and old Morisky scales was found in previous studies, but both had lower values than that of our study.

The known group comparison analysis indicated that the Malaysian version of the MMAS is a valid instrument for measuring medication adherences because the instrument was able to differentiate between patients who were clinically different. Glycaemic control represented by HbA1c was found to be significantly related with MMAS scores, in which lower HbA1c (better glycaemic control) was associated with higher adherence scores in the study cohort. This finding is accepted, as those patients with higher adherence have more awareness about the control of their diabetes and self-management processes, and they are concerned that poor adherence might worsen their clinical condition. Enhancement and improvement of patients' adherence may lead to improvement of their diabetes control. A significant association was found between the eight-item MMAS scores and diabetic control represented by HbA1c $\left(\chi^{2}=20.261 ; p \geq 0.001\right)$, with sensitivity and specificity of $77.6 \%$ and $45.3 \%$, respectively. The original eight-item MMAS shows a higher sensitivity and specificity [19] and this may be due to cultural differences and the manner in which the people answering the questions and patients may claim more adherence than is actually true. The Thailand study within the same patient group shows that their sensitivity and specificity [26] is lower than that of Morisky et al. [19]. However, sensitivity analysis using objective criteria or a gold standard test is needed to precisely answer this question. Unfortunately, the 
present study was limited in this respect. In addition, the data of this validation study was collected from one sitting and only from patients with diabetes type 2 and this might affect the ability to generalize the scale for other diseases.

\section{Conclusions}

The Malaysian version of the MMAS proved to be acceptable to patients; it is a simple questionnaire that can be administered by a trained nurse in face-to-face interviews to overcome non-response by those who cannot read, although the original questionnaire is a self-administered instrument. In summary, the findings from this validation study indicate that the Malaysian version of the MMAS is a reliable and valid measure of medication adherence because it shows acceptable test-retest reliability and convergent validity.

\section{Acknowledgments}

The study was funded by the research university Grant (1001/PFARMASI) from the Universiti Sains Malaysia. We would like to thank all participants for reviewing the questionnaire, for nurses in Penang General Hospital for their valuable help in data collection and patients for taking part in the study. All the authors declare that they have no competing or relevant conflict of interest to disclose.

\section{Abbreviations}

MMAS

DM

NHMS

Type 2 DM

HbA1c
Morisky Medication Adherence Scale

diabetes mellitus

National Health and Morbidity Survey

type 2 diabetes mellitus

haemoglobin A1C

\section{References}

1. International Diabetes Federation. Diabetes atlas. 3. Brussels, Belgium: International Diabetes Federation; 2006.

2. NHM III. The Third National and Morbidity Survey. 2006. Available at http://www.nih.gov.my/NHMS/abstracts_17.html

3. UK Prospective Diabetes Study Group. Intensive blood-glucose control with sulphonylureas or insulin compared with conventional treatment and risk of complications in patients with type 2 diabetes (UKPDS 33). Lancet. 1998; 352:837-53. [PubMed: 9742976]

4. Zimmet P, Alberti KG, Shaw J. Global and societal implications of the diabetes epidemic. Nature. 2001; 414:782-7. [PubMed: 11742409]

5. Rubin Richard R. Adherence to pharmacologic therapy in patients with Type 2 diabetes mellitus. Am J Med. 2005; 118:27S-34S. [PubMed: 15850551]

6. Rhee MK, Slocum W, Ziemer DC, Culler SD, Cook CB, El-Kebbi IM, et al. Patient adherence improves glycemic control. Diabetes Educ. 2005; 31:240-50. [PubMed: 15797853]

7. Krapek K, King K, Warren SS, George KG, Caputo DA, Mihelich K, et al. Medication adherence and associated hemoglobin A1C in type 2 diabetes. Ann Pharmacother. 2004; 38:1357-62.

[PubMed: 15238621]

8. World Health Organization. Report on medication adherence. Geneva: World Health Organization; 2003.

9. Cramer Joyce A. A systematic review of adherence with medications for diabetes. Diabetes Care. 2004; 27:1218-24. [PubMed: 15111553] 
10. Liebl A, Neiss A, Spannheimer A, Reitberger U, Wagner T, Gortz A. Costs of type 2 diabetes in Germany. Results of the CODE-2 study. Dtsch Med Wochenschr. 2001; 18:585-9. [PubMed: 11402924]

11. Osterberg L, Blaschke T. Adherence to medication. N Engl J Med. 2005; 353:487-97. [PubMed: 16079372]

12. Donnan PT, McDonald TM, Morris AD. Adherence to prescribed oral hypoglycemic medication in a population of patients with type 2 diabetes: a retrospective cohort study. Diabet Med. 2002; 19:279-84. [PubMed: 11942998]

13. Gordis L, Markowitz M, Lilienfeld AM. The inaccuracy in using interviews to estimate patient reliability in taking medications at home. Med Care. 1969; 7:49-54. [PubMed: 5804663]

14. Park LC, Lipman RS. A comparison of patient dosage deviation reports with pill counts. Psychopharmacology. 1964; 6:299-302.

15. Craig HM. Accuracy of indirect measures of medication compliance in hypertension. Res Nurs Health. 1985; 8:61-6. [PubMed: 3846318]

16. Grymonpre RE, Didur CD, Montgomery PR, Sitar DS. Pill count, self-report, and pharmacy claims data to measure medication adherence in the elderly. Ann Pharmacother. 1998; 32:749-54. [PubMed: 9681089]

17. Morisky DE, Green LW, Levine DM. Concurrent and predictive validity of a self-reported measurement of medication adherence and long-term predictive validity of blood pressure control. Med Care. 1986; 24:67-74. [PubMed: 3945130]

18. Shalansky SJ, Levy AR, Ignaszewski AP. Self-reported Morisky score for identifying nonadherence with cardiovascular medications. Ann Pharmacother. 2004; 38:1363-8. [PubMed: 15238622]

19. Morisky DE, Ang A, Krousel-Wood M, Ward H. Predictive validity of a medication adherence measure for hypertension control. J Clin Hypertens. 2008; 10:348-54.

20. Peat, JK. Health science research: a handbook of quantitative methods. Crows Nest: Allen \& Unwin; 2001.

21. Guillemin F, Bombardier C, Beaton D. Cross-cultural adaptation of health-related quality of life measures: literature review and proposed guidelines. J Clin Epidemiol. 1993; 46:1417-32. [PubMed: 8263569]

22. Wild D, Grove A, Martin M, Eremenco S, McElroy S, Verjee-Lorenzo A, et al. Principles of good practice for the translation and cultural adaptation process for patient-reported outcomes (PRO) measures: report of the ISPOR task force for translation and cultural adaptation. Value Health. 2005; 8:94-104. [PubMed: 15804318]

23. Bowling, A. Research methods in health: investigating health and health services. Buckingham: Open University Press; 2002.

24. Robinson, JP.; Shaver, PR.; Wrightsman, LS. Measures of personality and social psychological attitudes. San Diego, CA: Academic Press; 1991.

25. Cohen, JW. Statistical power analysis for the behavioural sciences. 2. Hillsdale, NJ: Lawrence Erlbaum Associates; 1988.

26. Sakthong P, Chabunthom R, Charoevisuthiwongs R. Psychometric properties of the Thai version of the 8-item Morisky Medication Adherence Scale in patients with type 2 diabetes. Ann Pharmacother. 2009; 43:950-7. [PubMed: 19366872]

27. Södergård B, Halvarsson M, Tully MP, Mindouri S, Nordström ML, Lindbäck S, et al. Adherence to treatment in Swedish HIV-infected patients. J Clin Pharm Ther. 2006; 31:605-16. [PubMed: 17176366]

28. Dawson, B.; Trapp, RG. Basic and clinical biostatistics. 4. McGraw Hill; 2004. International edition

29. Streiner, DL.; Norman, GR. Health measurement scales: a practical guide to their development and use. 2. New York: Oxford University Press; 1995. 
Table 1

Characteristic of patients according to the groups of adherence.

\begin{tabular}{|c|c|c|c|c|}
\hline Characteristics & Total sample $N=175$ & $\begin{array}{l}\text { Low adherence } N= \\
67(38.2 \%)\end{array}$ & $\begin{array}{l}\text { Medium adherence } N=78 \\
(44.5 \%)\end{array}$ & $\begin{array}{l}\text { High adherence } N=30 \\
(17.1 \%)\end{array}$ \\
\hline \multicolumn{5}{|l|}{ Age } \\
\hline Mean \pm SD & $60.56 \pm 9.16$ & $59.388 \pm 9.42$ & $60.96 \pm 8.54$ & $62.13 \pm 10.06$ \\
\hline \multicolumn{5}{|l|}{$\operatorname{Sex} N(\%)$} \\
\hline Male & $81(46.3)$ & $34(50.7)$ & $36(44.9)$ & $11(36.7)$ \\
\hline Female & $94(53.7)$ & $33(49.3)$ & $42(53.8)$ & $19(63.3)$ \\
\hline \multicolumn{5}{|l|}{$B M I$} \\
\hline Mean \pm SD & $26.58 \pm 5.02$ & $26.89 \pm 4.24$ & $26.41 \pm 5.76$ & $26.34 \pm 4.65$ \\
\hline \multicolumn{5}{|l|}{ Race N (\%) } \\
\hline Malay & $74(42.3)$ & $36(53.7)$ & $28(35.9)$ & $10(33.3)$ \\
\hline Chinese & $40(22.9)$ & $8(11.9)$ & $23(29.5)$ & $9(30.0)$ \\
\hline Indian & $59(33.7)$ & $22(32.8)$ & $27(34.6)$ & $10(33.3)$ \\
\hline Others & $2(1.1)$ & $1(1.5)$ & 0 & $1(3.3)$ \\
\hline \multicolumn{5}{|c|}{ Educational level $N(\%)^{a}$} \\
\hline No formal education & $22(12.6)$ & $15(22.4)$ & $6(7.7)$ & $1(3.3)$ \\
\hline Primary & $41(23.4)$ & $11(16.4)$ & $21(26.8)$ & $9(30.0)$ \\
\hline Secondary & $102(58.3)$ & $37(55.2)$ & $49(62.8)$ & $16(53.3)$ \\
\hline University & $10(5.7)$ & $4(6.0)$ & $2(2.6)$ & $4(13.3)$ \\
\hline \multicolumn{5}{|c|}{ Employment status $N(\%)$} \\
\hline Not employed & $66(37.7)$ & $24(35.8)$ & $32(41.0)$ & $10(33.3)$ \\
\hline Private & $24(13.7)$ & $11(16.4)$ & $8(10.3)$ & $5(16.7)$ \\
\hline Government & $35(20.0)$ & $17(25.4)$ & $15(19.2)$ & $3(10.0)$ \\
\hline Retired & $50(28.6)$ & $15(22.4)$ & $23(29.5)$ & $12(40.0)$ \\
\hline \multicolumn{5}{|c|}{ Diabetic duration (years) } \\
\hline Mean \pm SD & $10.54 \pm 7.47$ & $10.83 \pm 7.8$ & $10.02 \pm 6.97$ & $11.26 \pm 8.14$ \\
\hline \multicolumn{5}{|l|}{ Medications No. ${ }^{a}$} \\
\hline Mean \pm SD & $4.34 \pm 1.68$ & $4.45 \pm 1.8$ & $4.23 \pm 1.57$ & $4.4 \pm 1.73$ \\
\hline \multicolumn{5}{|l|}{$H b A 1 c^{a}$} \\
\hline Mean \pm SD & $7.95 \pm 1.56$ & $8.63 \pm 1.69$ & $7.74 \pm 1.33$ & $6.97 \pm 1.09$ \\
\hline \multicolumn{5}{|c|}{ Eight-item MMAS score ${ }^{a}$} \\
\hline Mean \pm SD & $6.13 \pm 1.72$ & $4.36 \pm 1.4$ & $6.94 \pm 0.53$ & $8.00 \pm 0.000$ \\
\hline \multicolumn{5}{|c|}{ Four-item original scale score ${ }^{a}$} \\
\hline Mean \pm SD & $2.43 \pm 1.24$ & $1.31 \pm 0.8$ & $2.87 \pm 0.9$ & $3.77 \pm 0.62$ \\
\hline
\end{tabular}

${ }^{a}$ Significant differences between groups. 
Table 2

Reliability test.

\begin{tabular}{|llcc|}
\hline & Mean \pm SD & Corrected item - total correlation & Cronbach's alpha if item deleted \\
\hline Question 1 & $0.56 \pm 0.49$ & 0.402 & 0.639 \\
Question 2 & $0.82 \pm 0.38$ & 0.402 & 0.637 \\
Question 3 & $0.80 \pm 0.4$ & 0.364 & 0.646 \\
Question 4 & $0.70 \pm 0.45$ & 0.344 & 0.653 \\
Question 5 & $0.96 \pm 0.19$ & 0.287 & 0.667 \\
Question 6 & $0.78 \pm 0.4$ & 0.461 & 0.621 \\
Question 7 & $0.69 \pm 0.46$ & 0.333 & 0.657 \\
Question 8 & $0.75 \pm 0.23$ & 0.459 & 0.641 \\
\hline
\end{tabular}

Cronbache's alpha was 0.675 for the total scale with significant intra-class correlation coefficient $(p<0.001)$. 
Table 3

The relationship between adherence categories and glycemic control groups. ${ }^{a}$

\begin{tabular}{|lllcl|}
\hline Glycemic group & Low adherence $($ score < 6) & Medium adherence $(\mathbf{6}<$ score $>8)$ & High adherence $($ score $=8)$ & $\boldsymbol{p}$ \\
\hline Good control HbA1c $<7 \%$ & $15(22.4)$ & $27(34.6)$ & $22(73.7)$ & $\leq 0.001$ \\
Poor control HbA1c $\geq 7 \%$ & $52(77.6)$ & $51(65.4)$ & $8(26.7)$ & $\leq 0.001$ \\
Total & $67(100)$ & $78(100)$ & $30(100)$ & \\
\hline
\end{tabular}

${ }^{a}$ Number (\%) of patients; $\chi^{2}=20.261, p \leq 0.001$. 